\title{
Incidencia poblacional del ácaro blanco (Polyphagotarsonemus latus, Banks) y otros artrópodos plagas en arreglos de policultivo y cultivo puro en Tisma, Nicaragua
}

\section{Broad mite (Polyphagotarsonemus latus, Banks.) population incidence and other arthropod pests in polyculture and pure crop arrangements in Tisma, Nicaragua}

Edgardo Jiménez-Martínez ${ }^{1}$, William Chamorro Aguilar ${ }^{2}$, Dirck Romero Lacayo ${ }^{3}$

1PhD en Entomología, Universidad Nacional Agraria, UNA, Profesor Titular, Docente-Investigador. E-mail: edgardo.jimenez@ci.una.edu.ni, Tel: $505-22632609^{2}{ }^{3}$ Ingenierio Agrónomo, UNA.

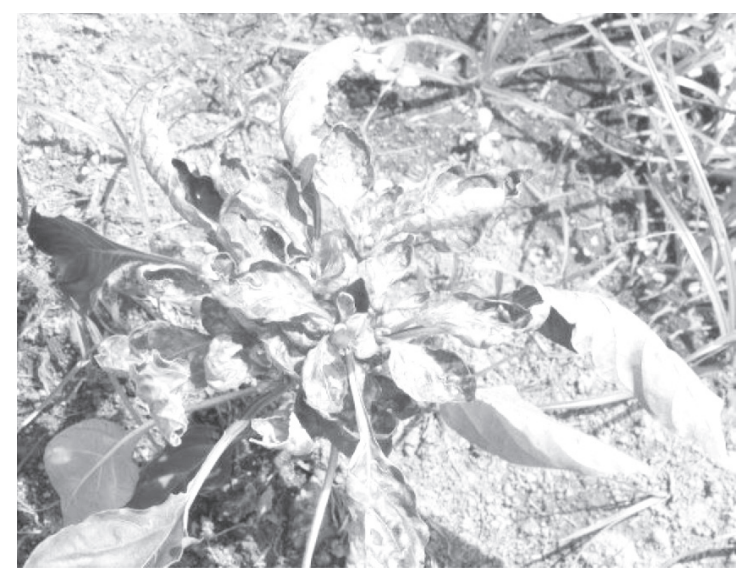

\section{RESUMEN}

Los policultivos juegan un papel importante en la biodiversidad de un agroecosistema, estos promueven servicios ecológicos tales como reciclaje de nutrientes, control biológico de plagas, enfermedades y arvenses, incremento en la producción de biomasa, conservación de suelos y agua, mejoramiento de calidad físico-química del suelo, reducción de la toxicidad al evitar o disminuir el uso de pesticidas. El estudio se realizó en el municipio Tisma, Masaya en la finca "El Chagüite" en los meses de Septiembre a Noviembre del 2014, con el objetivo de estudiar el efecto de dos sistemas de siembra, cultivo puro y policultivo, sobre la ocurrencia poblacional de artrópodos plagas y benéficos e incidencia poblacional del ataque del ácaro blanco en el cultivo de chiltoma. Para el desarrollo de la investigación se establecieron cuatro parcelas, una con el arreglo en policultivos (chiltoma, maíz, tomate, yuca y quequisque) y los restantes se establecieron con cultivo puro de chiltoma, maíz y tomate. Las variables evaluadas semanalmente fueron la ocurrencia poblacional de artrópodos plagas y benéficos, ocurrencia del ácaro blanco y el rendimiento obtenido en los cultivos de chiltoma, maíz y tomate para el análisis del uso equivalente de la tierra (UET). En general se observó una tendencia de menor ocurrencia de plagas, menor densidad poblacional de ácaro blanco, mayor densidad poblacional de artrópodos benéficos y un mejor aprovechamiento de la tierra por unidad de área en el sistema de siembra de policultivo. Palabras clave: Cultivo puro, policultivo, chiltoma, ácaro blanco.

\begin{abstract}
Polycultures play an important role in the biodiversity of agroecosystems, they promote ecological services such as nutrient cycling, biological control of pests, diseases and weeds, increased biomass production, soil and water conservation, improvement physical-chemistry soil properties, reduced toxicity by avoiding the over use of pesticides. This study was conducted in the municipality of Tisma, Masaya in the farm "El Chagüite" in the months of September to November 2014, in order to compare two planting systems, monoculture and polyculture, on the population of pests and beneficial arthropods; as well as the severity of the attack of broad mite in sweet pepper. For the development of this research, four experimental plots were arranged; one with the arrangement of sweet pepper, corn, cassava, quequisque, and tomato; the other three were established in monoculture of sweet pepper, corn and tomato respectively. The weekly evaluated variables were, the population incidence of pests and beneficial arthropods present in the culture of sweet pepper. Broad mites incidence and severity present in the cultivation of sweet pepper were also recorded. Additionally, the production of sweet pepper, corn, and tomato were harvested in order to calculate the land equivalent ratio (LER). A general trend of lower occurrence of pests, lower population density of white mite, higher population density of beneficial arthropods and better land use per unit area in polyculture planting system was observed.
\end{abstract}

Keywords: Monoculture, polyculture, sweet pepper, broad mite.
Recibido: 25 de octubre 2016

Aceptado: 21 de marzo del 2017
Los artículos de la revista La Calera de la Universidad Nacional Agraria, Nicaragua, se comparten bajo términos de la licencia Creative Commons: Reconocimiento, No Comercial, Compartir Igual. Las autorizaciones adicionales a las aquí delimitadas se pueden obtener en el correo freddy.aleman@ci.una.edu.ni Copyright 2017. Universidad Nacional Agraria 


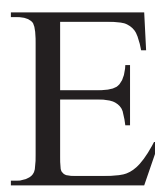
$\mathrm{n}$ la actualidad, diversas investigaciones han demostrado que la biodiversidad en un agroecosistema es esencial para el manejo de insectos plagas. Las poblaciones insectiles pueden ser estabilizadas mediante el diseño y construcción de arquitecturas vegetales que sustenten las poblaciones de artrópodos benéficos o que tengan efectos disuasivos directos sobre los insectos plagas (Altieri y Nicholls, 2000).

Los policultivos juegan un papel importante en la biodiversidad de un agroecosistema, estos promueven los servicios ecológicos presentes en el agroecosistema como reciclaje de nutrientes, control biológico de plagas, enfermedades y arvenses, incremento en la producción de biomasa, conservación de suelos y agua, mejoramiento de calidad físico-química del suelo, reducción de la toxicidad en el agroecosistema al evitar o disminuir el uso de pesticidas e incrementar y sustentar la producción agrícola a largo plazo. (Altieri y Nicholls, 2000).

El cultivo puro se basa en sembrar el mismo cultivo en cierta área de tierra por un período de tiempo determinado. El uso del cultivo puro facilita la siembra y cosecha en grandes extensiones de tierra. Además de facilitar el uso de maquinaria agrícola, reduciendo así la mano de obra utilizada en el manejo del cultivo. También, el cultivo puro fomenta el uso de fertilizantes y pesticidas sintéticos, lo que en cierto grado facilita las labores de fertilización y manejo de plagas y enfermedades. Sin embargo, el cultivo puro acarrea consigo una serie de problemas desde el punto de vista social, económico y ecológico, fomenta la pobreza en el área rural, disminuye la mano de obra utilizada en la producción e imposibilita una competencia leal entre grandes y pequeños productores (GRACE Communications Foundation, 2015).

Laguna et al. (2006) mencionan que los principales artrópo dos plagas de la chiltoma son el picudo de la chiltoma (Anthonomus eugenii Cano) y el ácaro blanco (Polyphagotarsonemus latus Banks), los que han llegado a causar pérdidas de hasta un $100 \%$ en el fruto de chiltoma. Dichos autores recomiendan diversas prácticas para el manejo integrado de plagas en chiltoma, dentro de las cuales se encuentra el uso de policultivos. Se conoce como policultivos al uso de dos o más cultivos en la misma área productiva, dando lugar a una competencia interespecífica o una complementación entre los cultivos. (Altieri y Nicholls, 1999).

Lo descrito anteriormente ha sido comprobado por trabajos investigativos realizados por Ayala (1992), Garache y López (2007) y García y Angulo (2008); quienes realizaron investigaciones, evaluando la ocurrencia poblacional de artrópodos plagas y benéficos en asocios de policultivo y cultivo puro. Concluyendo que la mayor ocurrencia de artrópodos benéficos y menor ocurrencia de artrópodos plagas se da en los asocios de cultivo. Tomando en cuenta lo expuesto anteriormente, este trabajo está orientado a determinar los efectos de los arreglos de siembra cultivo puro (chiltoma) y policultivo (maíz, chiltoma, quequisque, yuca y tomate) sobre la ocurrencia poblacional de artrópodos plagas y benéficos en el municipio de Tisma, Masaya.

\section{MATERIALES Y MÉTODOS}

Ubicación del ensayo. El estudio se llevó a cabo en el municipio de Tisma departamento de Masaya, ubicado a $36 \mathrm{~km}$ de la parte norte de la capital Managua. Entre las coordenadas $12^{\circ} 04^{\prime}$ latitud norte y $86^{\circ} 01^{\prime}$ longitud oeste y a una altura de $50 \mathrm{msnm}$. Tisma limita al norte con Tipitapa, al suroeste con el departamento de Masaya y al este con el departamento de Granada. Posee una superficie de 126.17 km2 con una densidad poblacional de 12,697 habitantes que se distribuyen en la zona urbana en un $33.42 \%$ y la zona rural en un $66.57 \%$. Su clima se caracteriza como tropical de sabana, con temperaturas de $27.5^{\circ} \mathrm{C}$ y precipitaciones pluviales anuales entre los 1,200 y 1,400 mm. (AMUNIC, 2005). El presente trabajo se estableció en la finca El Chagüite propiedad de la agricultora Elizabeth González.

Material genético de los cultivos utilizados en el experimento. El material genético se seleccionó de acuerdo a los cultivares más utilizados por los productores de la zona Chiltoma (Híbrido Nathalie), Maíz (NBS), Yuca (Reyna), Quequisque (Criollo), Tomate (Shanty).

Arreglo de las parcelas. Para el arreglo de siembra cultivo puro, se contó con tres parcelas. Una parcela de chiltoma, una de maíz y una de tomate. Debido a que los cultivos de yuca y quequisque son perennes, no se establecieron parcelas de cultivo puros de estos. Cada parcela contaba con un área total $252 \mathrm{~m} 2(36 \mathrm{~m} * 7 \mathrm{~m})$. Las parcelas útiles fueron de $132.3 \mathrm{~m} 2$ $(31.5 \mathrm{~m} * 4.2 \mathrm{~m})$. Distancias de siembra para cultivo puro de chiltoma y tomate: entre plantas $0.5 \mathrm{~m}$, entre surcos 1.4 $\mathrm{m}$ para una densidad poblacional de 189 plantas/parcela útil. Distancia de siembra para el cultivo puro de maíz: entre surcos $0.7 \mathrm{~m}$, entre plantas 0.25 para una densidad poblacional de 756 plantas/parcela útil. El sistema de siembra policultivo poseía un área total de $1,058.4 \mathrm{~m} 2(36 \mathrm{~m} * 29.4 \mathrm{~m})$ y una parcela útil de $749.7 \mathrm{~m} 2(31.5 \mathrm{~m} * 23.8 \mathrm{~m})$. El arreglo de los cultivos era para maíz con 4 surcos, chiltoma 6 surcos, Yuca, Quequisque y Tomate 3 surcos. Distancias de siembra usadas en arreglo policultivo de chiltoma: entre plantas 0.5 , entre surcos $1.4 \mathrm{~m}$, para una densidad poblacional de 378 plantas/ parcela útil. Distancia de siembra para el cultivo de maíz en policultivo: entre surcos $0.7 \mathrm{~m}$, entre plantas $0.25 \mathrm{~m}$, para una densidad poblacional de 504 plantas/parcela útil.

Muestreo de artrópodos. La toma de datos se realizó en el cultivo de chiltoma en ambas parcelas, esta se realizó semanalmente en horas de la mañana, se seleccionaron cinco puntos al azar con veinte plantas para un total de 100 plantas; las cuales fueron evaluadas permanentemente a lo largo del estudio. En cada planta se evaluó las hojas, botones florales y frutos. (Garache y López, 2007). Para el muestreo de ácaro blanco se utilizó una lupa de Steinheil con $16 \mathrm{X}$ de aumento. (Laguna et al. 2006).

Variables evaluadas. Artrópodos Plagas. Los artrópodos plagas determinados fueron: número de ácaros blancos por planta (Polyphagotarsonemus latus Banks), número de mosca blanca por planta (Bemisia tabaci Gennadius), número de áfidos por planta (Aphis gossypii, Myzus persicae), y úmero 
de minador de las hojas por planta (Liriomyza sativae Blanchard)- Los artrópodos Benéficos determinados fueron: número de abejas por planta (Apis mellifera L.), número de arañas por planta (Orden: Araneae), número de mariquitas por planta (Coccinela septempunctata L.), úmero de hormigas por planta (Solenopsis spp.).

Uso equivalente de la tierra. Gliessman (2002) explica que el uso equivalente de la tierra es una herramienta muy útil para evaluar los rendimientos obtenidos en sistemas de asocios de cultivos en contraste con sistemas de cultivo puro. Para calcular el uso equivalente de la tierra se emplea la siguiente fórmula: UET $=\sum$ Ypi/Ymi, donde, UET $=$ Uso equivalente de la Tierra, $\sum=$ Sumatoria, Ypi $=$ Rendimiento en sistemas en asocio (Kg/ha) y Ymi = Rendimiento en cultivo solo (Kg/ha). Gliessman (2002) menciona que un valor de UET igual a 1.0, indica que no existen diferencias entre rendimientos de los sistemas de cultivos evaluados. En cambio si se obtiene un valor mayor a 1.0, este indica que existe una ventaja para el sistema de asocio de cultivos. Esto quiere decir que existe una interferencia positiva entre los cultivos que componen el asocio, así como también cualquier competencia interespecífica no es tan negativa para el asocio en comparación con la del cultivo puro.

Análisis estadístico de los datos. A cada una de las variables registradas en el experimento se les realizó un análisis de varianza con un nivel de significancia del 95\%. Posteriormente se realizó una separación de medias mediante Tukey con el programa estadístico SAS (SAS, 2003).

\section{RESULTADOS Y DISCUSIÓN}

Los principales artrópodos plagas y benéficos encontrados en este estudio se detallan en el cuadro 1. Las poblaciones más numerosas de artrópodos plagas correspondían a ácaro blanco (Polyphagotarsonemus latus Banks), mosca blanca (Bemisia tabaci Gennadius), áfidos (Aphididae), minador de la hoja (Lyriomiza sativae Blanchard) y picudo de la chiltoma (Anthonomus eugenii Cano). No se encontraron individuos de gusano del fruto (Heliothis spp, Spodoptera spp). La población de picudo de la chiltoma no pudo ser analizada estadísticamente debido a que esta era muy baja. Las poblaciones más numerosas de artrópodos benéficos fueron las de arañas (Araneae), hormigas (Solenopsis spp.), abejas (Apis mellifera L.) y mariquitas (Coccinella septempunctata L.).
Número total de artrópodos plagas y benéficos en chiltoma. El sistema de cultivo puro presentó un total de 5388 insectos plagas, y 130 insectos benéficos. En cambio en el sistema de policultivo se encontraron 3797 insectos plagas y 22 insectos benéficos. La población de artrópodos plagas fue mayor para el sistema cultivo puro. El arreglo de siembra policultivo presentó la mayor cantidad de la entomofauna benéfica.

El cuadro 2 muestra que el sistema de siembra cultivo puro presentó 1.35 artrópodos plagas por planta. Resultando ser el arreglo de siembra con la mayor densidad poblacional de herbívoros presentes en este estudio. El arreglo de siembra policultivo registró 0.06 artrópodos benéficos en promedio por planta, siendo el sistema con la mayor ocurrencia de entomofauna benéfica.

Estos resultados coinciden con los obtenidos en estudios similares donde también se evaluó la influencia de arreglos de siembra cultivo puro y policultivo de diferentes cultivares sobre las densidades poblacionales de artrópodos plagas y benéficos. Ayala (1992), obtuvo 1.8 artrópodos plagas y 0.12 artrópodos benéficos por planta en cultivo puro y 0.2 herbívoros y 0.50 artrópodos benéficos por planta en policultivo. Garache y López (2007) obtuvieron un total de 5,063 artrópodos plagas y 198 artrópodos benéficos para cultivo puro; para el sistema policultivo contabilizaron un total de 1,675 artrópodos plagas y 510 artrópodos benéficos. García y Angulo (2008) totalizaron 2,956.39 artrópodos plagas y 147.55 artrópodos benéficos en cultivo puro, en el sistema policultivo observaron 2,584.54 artrópodos plagas y 333.6 artrópodos benéficos.

Cuadro 2. Niveles poblacionales de artrópodos plagas y benéficos en chiltoma en cultivo puro y policultivo en Tisma, Masaya, Septiembre-noviembre, 2014

\begin{tabular}{lcc}
\hline Variables & Plagas & Benéficos \\
\hline Tratamientos & Media $\pm \mathrm{ES}$ & Media $\pm \mathrm{ES}$ \\
\hline Cultivo puros & $1.35 \pm 0.03 \mathrm{a}$ & $0.03 \pm 0.0039 \mathrm{~b}$ \\
Policultivo & $0.95 \pm 0.03 \mathrm{~b}$ & $0.06 \pm 0.0039 \mathrm{a}$ \\
CV $(\%)$ & 166.83 & 557.61 \\
P & $<0.0001^{*}$ & $<0.0001^{*}$ \\
F; Ft; df; $\mathrm{n}$ & $85.87 ; 3.85 ; 7998 ; 8000$ & $17.58 ; 3.85 ; 7998 ; 8000$
\end{tabular}

CV: Coeficiente de variación, E.S: Error estándar de la media, P: Probabilidad calculada por InfoStat v. 2015, F: Fisher calculado, Ft: Fisher tabulado al 5\%, df: grados de libertad del error, n: Número de datos utilizados, *: Estadísticamente significativo, NS: No significativo estadísticamente.

Cuadro 1. Artrópodos plagas y benéficos en chiltoma en cultivo puro y policultivo en Tisma, Masaya, Septiembre-noviembre, 2014

\begin{tabular}{|c|c|c|c|c|c|c|}
\hline Antrópodo & $\begin{array}{c}\text { lo Nombre } \\
\text { Común }\end{array}$ & Orden & Familia & $\begin{array}{l}\text { Nombre } \\
\text { científico }\end{array}$ & Categoría & $\begin{array}{c}\mathrm{N}^{\mathrm{o}} \text { de } \\
\text { indidividuos }\end{array}$ \\
\hline \multirow[t]{5}{*}{ Plagas } & Ácaro blanco & Trombidiformes & Tarsonemidae & $\begin{array}{l}\text { Polyphagotarsonemus } \\
\text { latus }\end{array}$ & $\begin{array}{l}\text { Raspador/ } \\
\text { Chupador }\end{array}$ & 3932 \\
\hline & Mosca blanca & Hemíptera & Aleyrodidae & Bemisia tabaci & Chupador & 2617 \\
\hline & Áfidos & Hemíptera & Aphididae & ------------- & Chupador & 1394 \\
\hline & Minador de la hoja & Díptera & Agromycidae & Lyriomiza sativae & Minador & 1233 \\
\hline & Picudo & Coleóptero & Curculionidae & Anthonomus eugenii & Masticador & 9 \\
\hline \multirow[t]{4}{*}{ Benéficos } & Araña & Araneae & Aracnidae & -------------- & Depredador & 187 \\
\hline & Hormiga & Hymenóptera & Formicidae & Solenopsis spp & Depredador & 123 \\
\hline & Abeja & Hymenóptera & Apidae & Aphis mellifera & Polinizador & 25 \\
\hline & Mariquita & Coleóptera & Coccinellidae & Coccinella septempun & ata Depredador & 17 \\
\hline
\end{tabular}


Ocurrencia poblacional ácaro blanco, mosca blanca, áfidosy minador de la hoja. El cuadro 3 muestra la mayor ocurrencia de ácaro blanco de 2.16 registrada en el sistema cultivo puro. El sistema policultivo presentó la menor densidad de ácaro blanco con 1.77 ácaro blanco por planta. El CATIE (1993) menciona que el ácaro blanco está presente durante todo el ciclo de vegetativo de la chiltoma. Sin embargo, este ataca principalmente los primeros estados vegetativos de la planta. El ciclo de vida del ácaro blanco, está altamente influenciado por la temperatura ambiental y la humedad relativa, al presentarse temperaturas de $30^{\circ} \mathrm{C}$ con humedad relativa de $70 \%$ el ciclo de vida dura 3 días. Debido a este alto potencial reproductivo, el ácaro blanco puede alcanzar densidades poblacionales perjudiciales en poco tiempo.

Liebman (1999) explica que al existir una mejor circulación de aire en los policultivos, la humedad ambiental es disminuida. Pino et al. (1999) mencionan que al existir una reducción de la intensidad lumínica que entra al agroecosistema, se da una disminución de la temperatura del aire. Esto conlleva a la creación de un microclima característico de los policultivos. Al contar con temperaturas menores, el policultivo influye en la tasa de reproducción y duración del ciclo de vida del ácaro blanco. Lo que es demostrado al obtener una menor ocurrencia poblacional de dicho artrópodo en el asocio de cultivo.

El análisis de varianza realizado a la ocurrencia poblacional de mosca blanca, demuestra que el sistema cultivo puro presentó el mayor número de individuos, 1.89 moscas blancas por planta. En el sistema policultivo se registraron 0.72 moscas blancas por planta (cuadro 3). Según el CATIE (1993) el ciclo de vida de la mosca blanca dura alrededor de 21 días. Siendo su punto crítico de infección las primeras 5 semanas de vida del cultivo de chiltoma. Mau y Martin (2007) explican que el rango de temperatura en el que

la mosca blanca puede desarrollarse de manera normal es de $10^{\circ} \mathrm{C}$ a $32{ }^{\circ} \mathrm{C}$, siendo $27^{\circ} \mathrm{C}$ la temperatura óptima. Mau y Martin (2007) explica que los individuos de mosca blanca son atraídos por el color amarillo. Además, estas son atraídas por hojas con bastante pilosidad. El cultivo del tomate fue utilizado dentro del sistema policultivo. Este cumplió una función de cultivo trampa para mosca blanca debido a que sus hojas son pilosas contrario a las hojas glabras de chiltoma. Además. Las flores del tomate son de color amarillo, en contraste a las flores blancas de chiltoma. Lo que conllevó a que las moscas blancas fuesen atraídas por el cultivo de tomate. Dando como resultado una menor densidad poblacional de este insecto en el asocio de cultivos.

El análisis de varianza realizado para las poblaciones de áfidos indicó que el sistema cultivo puro presenta la mayor población de áfidos con un promedio de 0.82 por planta en comparación a los 0.57 áfidos por planta en policultivo. (cuadro 3). El ciclo de vida y la tasa de reproducción de los áfidos, es afectada por condiciones ambientales tales como temperatura, precipitación y humedad relativa. (CATIE, 1993). Temperaturas bajas y altas humedades relativas dificultan la formación de colonias de áfidos en el cultivo de chiltoma. Esto se evidencia en el sistema policultivo, en el cual la población de áfidos es menor en comparación con el sistema cultivo puro debido a la presencia del microclima existente en el asocio de cultivo.

El análisis de varianza realizado para las poblaciones de minador de la hoja demostró que el sistema policultivo mostró la mayor cantidad de galerías de minador de la hoja con una media 0.72 galerías mientras que el sistema cultivo puro presentó una media de 0.51 galerías por planta. (cuadro 3). El minador de la hoja logra completar su ciclo biológico en aproximadamente 15 días con una temperatura de $30^{\circ} \mathrm{C}$. El minador de la hoja posee muchas plantas hospedantes; dentro de las que se encuentran las especies pertenecientes a la familia Solanaceae. (Capinera, 2001). Al contar con temperaturas más bajas, el sistema policultivo propiciaba el desarrollo del minador de la hoja. Además, el sistema policultivo contaba con dos plantas de la familia Solanaceae, tomate y chiltoma. Lo que justifica la mayor densidad poblacional de minadores de la hoja en el sistema policultivo.

Cuadro 3. Niveles poblacionales de ácaro blanco, mosca blanca, áfidos y minador de la hoja en chiltoma en cultivo puro y policultivo en Tisma, Masaya, Septiembre-noviembre, 2014

\begin{tabular}{lcccc}
\hline Variables & Ácaro blanco & Mosca blanca & Áfidos & Minador de la hoja \\
& & & & \\
\hline Tratamientos & Media + ES & Media + ES & Media + ES & Media + ES \\
& & & & \\
\hline Cultivo puro & $2.16+0.08 \mathrm{a}$ & $1.89+0.07 \mathrm{a}$ & $0.82+0.05 \mathrm{a}$ & $0.51+0.03 \mathrm{a}$ \\
Policultivo & $1.77+0.08 \mathrm{~b}$ & $0.72+0.04 \mathrm{~b}$ & $0.57+0.05 \mathrm{~b}$ & $0.72+0.03 \mathrm{~b}$ \\
CV. & 126.68 & 138.04 & 233.38 & 171.28 \\
P & $0.0005^{*}$ & $<0.0001^{*}$ & $0.0008^{*}$ & $<0.0001^{*}$ \\
F; Ft; df; $\mathrm{n}$ & $12.01 ; 3.85 ; 1998 ; 2000$ & $210.13 ; 3.85 ; 1998 ; 2000$ & $11.25 ; 3.85 ; 1998 ; 2000$ & $20.73 ; 3.85 ; 1998 ; 2000$
\end{tabular}

CV: Coeficiente de variación, E.S: Error estándar de la media, P: Probabilidad calculada por InfoStat v.2015, F: Fisher calculado, Ft: Fisher tabulado al 5\%, df: grados de libertad del error, n: Número de datos utilizados, *: Estadísticamente significativo, NS: No significativo estadísticamente.

Ocurrencia poblacional de abejas, arañas, mariquitas y hormigas. El análisis de varianza indica que el sistema policultivo posee la mayor cantidad de insectos benéficos (Cuadro 4). La mayor cantidad de abejas se encuentra en el sistema policultivo con un promedio de 0.02 individuos por planta y el sistema cultivo puro cuenta con la menor cantidad de abejas en promedio, con un aproximado de 0.001 abejas por planta.

El análisis de varianza realizado para las poblaciones de arañas indicó que el sistema policultivo presenta la mayor presencia con un promedio de 0.10 arañas por planta en comparación con 0.90 arañas por planta en cultivo puro. La mayor presencia de mariquitas y hormigas las presento el sistema policultivo con 0.01 y 0.09 y en menor presencia el sistema de cultivo puro con 0.004 mariquitas por planta y 0.03 hormigas por planta. 
Cuadro 4. Niveles poblacionales de abejas, arañas, mariquitas y hormigas en chiltoma en cultivo puro y policultivo en Tisma, Masaya, Septiembre-noviembre, 2014

\begin{tabular}{lcccc}
\hline Variables & Abejas & Arañas & Mariquitas & Hormigas \\
\hline Tratamientos & Media + ES & Media + ES & Media + ES & Media + ES \\
\hline Cultivo puro & $0.001+0.0042 \mathrm{~b}$ & $0.09+0.01$ & $0.004+0.0035$ & $0.03+0.01 \mathrm{~b}$ \\
Policultivo & $0.02+0.0042^{\mathrm{a}}$ & $0.10+0.01$ & $0.01+0.0035$ & $0.09+0.01 \mathrm{a}$ \\
CV. & 1050.07 & 352.84 & 1311.11 & 505.62 \\
P & $0.0001^{*}$ & $0.4560 \mathrm{NS}$ & $0.0711 \mathrm{NS}$ & $<0.0001^{*}$ \\
F; Ft; df; $n$ & $15.35 ; 3.86 ; 1998 ; 2000$ & $0.56 ; 3.86 ; 1998 ; 2000$ & $3.26 ; 3.86 ; 1998 ; 2000$ & $23.21 ; 3.96 ; 88 ; 90$
\end{tabular}

CV: Coeficiente de variación, E.S: Error estándar de la media, P: Probabilidad calculada por InfoStat v.2015, F: Fisher calculado, Ft: Fisher tabulado al 5\%, df: grados de libertad del error, n: Número de datos utilizados, *: Estadísticamente significativo, NS: No significativo estadísticamente.

Uso equivalente de la tierra (UET). Para el cálculo del uso equivalente de la tierra se tomó el rendimiento de tres cultivos, chiltoma, maíz y tomate, debido a que los otros dos cultivos, yuca y quequisque, se encontraban aún en crecimiento vegetativo (cuadro 5).

Cuadro 5. Uso equivalente de la tierra para los cultivos de chiltoma, maíz y tomate cultivo puro y policultivo en Tisma, Masaya, Septiembre-noviembre, 2014

\begin{tabular}{lccc}
\hline Cultivo & $\begin{array}{c}\text { Rendimiento en } \\
\text { policultivo }(\mathrm{kg} / \mathrm{ha})\end{array}$ & $\begin{array}{c}\text { Rendimiento en } \\
\text { cultivo puro }(\mathrm{kg} / \mathrm{ha})\end{array}$ & UET parcial \\
\hline Chiltoma & 14.98 & 16.57 & 0.9 \\
Maíz & 1033.42 & 2587.79 & 0.4 \\
Tomate & 220.89 & 232.47 & 0.68 \\
UET & & & 1.99 \\
\hline
\end{tabular}

La ocurrencia poblacional del acaro blanco en el sistema policultivo fue menor en comparación al sistema cultivo puro.

La población de artrópodos plagas fue mayor en los arreglos de cultivo puro comparado con los policultivos.

Las poblaciones de artrópodos benéficos fueron mayores en el sistema policultivo comparado con los sistemas en cultivo puro.

El análisis del uso equivalente de la tierra (UET) obtenido en este estudio es de 1.99, es decir que se necesitaría aproximadamente el doble de área en cultivo puros para obtener la misma producción de un sistema en policultivo.
Gliessman (2002) expresa que cuando se obtienen resultados del uso equivalente de la tierra por encima de 1.0, indica la existencia de una interferencia interespecífica positiva entre los cultivos que conforman el asocio. Es decir, que el conjunto de cultivos crea condiciones que favorecen al sistema. Dichas condiciones, evidenciadas en este estudio, son la presencia del microclima, característico del policultivo, y la menor ocurrencia de artrópodos herbívoros en los cultivos en asocio.

\section{AGRADECIMIENTO}

Los autores de esta investigación agradecen a la Sra. Elizabeth González y al Sr. Anuar González, productores de Tisma, Masaya por prestarnos su finca donde se realizó la investigación, a la Universidad Nacional Agraria (UNA) por la financiación económica de este estudio.

\section{REFERENCIAS BIBLIOGRÁFICAS}

Altieri, MA; Nicholls CI. 1999. Biodiversidad y manejo de plagas en agro ecosistemas. Trad. MA Altieri. Barcelona, ES, Icaria. 245 p. (Perspectivas Agroecológicas $\mathrm{N}^{\circ} 2$ ).

Altieri, MA; Nicholls CI. 2000. Agroecología. México D.F., MX, PNUMA. 250 p. (Textos Básicos para la Formación Ambiental).

AMUNIC (Asociación de Municipios de Nicaragua). 2005. Municipios: Caracterización de municipios de Masaya (en línea). Managua, NI. Consultado 15 oct. 2014. Disponible en http//www.amunic.org/

Ayala, O. 1992. Efecto de policultivo repollo-tomate sobre la entomofauna del cultivo de repollo. Tesis de graduación. Managua NI, UNA. $51 \mathrm{p}$.

Capinera, JL. 2001. Vegetable leafminer. University of Florida. Consultado 6 ago. 2015. Disponible en http://entnemdept.ufl.edu/ creatures/veg/leaf/vegetable_leafminer.htm

Centro Agronómico Tropical de Investigación y Enseñanza (CATIE). 1993. Guía para el manejo integrado de plagas del cultivo de chile dulce. Turrialba, CR. 143 p.

Gliessman, SR. 2002. Agroecología: Procesos Ecológicos en Agricultura Sostenible. Eds. E Rodríguez, T Benjamín, L Rodríguez, A Cortés. Trads. R Cohen, A González-Jácome, JJ Jiménez Osornio et al. Turrialba, CR, CATIE. 359 p. 
Garache Guido, MA; López López, GR. 2007. Efectos de policultivo tomate (Lycopersicum esculentum Mill), chiltoma (Capsicum annuит L) y maíz (Zea mays L) en la ocurrencia poblacional de insectos plagas y artrópodos beneficos y el uso equivalente de la tierra Tisma-Masaya. Tesis Ing. Agr. Managua, NI, UNA. 75 p.

García Guevara, KA.; Angulo Rivas, L.K. 2008. Efecto de cultivos en asocio Pepino (Cucumis sativus L.), Pipian (Cucurbita pepo L.) y frijol de vara (Vigna unguiculata L. walp), en la ocurrencia poblacional de insectos plagas, benéficos y el rendimiento en Tisma, Masaya (Tesis Ingeniero en Sanidad Vegetal), Universidad Nacional Agraria, UNA).

GRACE Communications Foundation. 2015. Industrial Crop Production. Consultado 26 feb. 2015. Disponible en http://www.sustainabletable.org/804/industrial-crop-production.

Laguna, T; Gutiérrez, C; Sarria, M. 2006. Guía Tecnológica de Chiltoma. Ed. H Obregón. Managua, NI, Instituto Nicaragüense de Tecnología Agropecuaria. 44 p.

Liebman, M. 1999. Sistemas de Policultivos. In Altieri, MA. Agroecología: Bases científicas para una agricultura sustentable. Montevideo, UY, Nordan-Comunidad. P 191-202.

Mau, RF; Martin Kessing, JL. 2007. Bemisia tabaci (Gennadius). Consultado 6 ago. 2015. Disponible en http://www.extento.hawaii. edu/kbase/crop/type/b_tabaci.htm

Pino, MA; Terry, E; A, L; P, M; F, S. 2000. Respuesta de las plantas de tomate a la modificación de algunas variables del microclima en un sistema protegido con sombra natural. En Cultivos Tropicales 21 (2): 33-36. Consultado 6 ago. 2015. Disponible http://www. redalyc.org/pdf/1932/193215024006.pdf.

SAS Institute, 2003. University of Nebraska. Cary, NC, USA.V.91. 